\title{
LA IMPORTANCIA DE LA ERGONOMÍA PARA LOS PROFESIONALES DE LA SALUD
}

\author{
THE IMPORTANCE OF ERGONOMICS FOR HEALTH WORKERS
}

\author{
ELÍAS APUD, PhD.* y FELIPE MEYER, MSc. **
}

\begin{abstract}
RESUMEN
La ergonomía es una multidisciplina preocupada de la adaptación del trabajo al hombre. Su desarrollo es reciente en nuestro medio, existiendo una gran necesidad de que los profesionales del área de la salud incorporen criterios ergonómicos en sus actividades, ya que en el mundo moderno existe un conjunto de patologías que pueden ser desencadenas o agravadas por el trabajo. En estos casos, los tratamientos no son efectivos si no se corrigen las causas que los generan. El artículo presenta una visión conceptual de ergonomía, sus objetivos, campos de acción y los aspectos fisiológicos, psicológicos, biomecánicos, ambientales y organizacionales que la sustentan, destacando su carácter multidisciplinario. Se analiza también sus proyecciones y su utilidad tanto en la adaptación de métodos tradicionales de trabajo como en las tecnologías del futuro.
\end{abstract}

Palabras claves: Ergonomía, trabajadores de la salud, carga física, carga mental.

\begin{abstract}
Ergonomics is a multidiscipline preoccupied of the adaptation of work to man. This concept is fairly new in our country, but it is very necessary that professionals who take care of health of the population get fundamentals of this discipline. This is because in modern world there are a number of pathologies which are caused or aggravated by work. In those cases, treatments are not effective if actions are not taken to correct the causes that generates the symptoms. The article presents an overview of Ergonomics, its objectives, field of action and the physiological, psychological, biomechanical, environmental and organizational aspects which constitutes its basis. A discussion is also made on the projections of Ergonomics and its utility in the adaptation of traditional work methods as well as in technologies of the future.
\end{abstract}

Keywords: Ergonomics; health workers; physical work load; mental load.

Recepcionado: 20.06.2003. Aceptado: 25.06.2003.

\section{INTRODUCCIÓN}

Los profesionales del área de la salud atienden trabajadores que, en muchos casos, presentan patologías derivadas de sus condiciones de trabajo. Por ejemplo, en el mundo moderno el estrés laboral, los síntomas músculo-esqueléticos asociados a trabajo repetitivo, posturas inadecuadas y manejo manual de materiales, la obesidad vinculada a trabajo sedentario, la fatiga crónica, etc., podrían disminuir o aminorarse con un adecuado diseño del trabajo. Desde este punto de vista, es importante incluir en la formación de los profesionales de la salud conceptos de ergonomía, ya que, si los agentes causales persisten en el medio ambiente laboral, los tratamientos no serán efectivos y los trabajadores seguirán reiterando síntomas que les alteran su bienestar físico y mental. Aun cuando el primer laboratorio de ergonomía del país se crea en 1972 en la Universidad de Concep-

*Profesor Titular Unidad de Ergonomía, Facultad de ciencias Biológicas, Universidad de Concepción. eapud@udec.cl

**Colaborador acádemico Unidad de Ergonomía, Facultad de Ciencias Biológicas, Universidad de Concepción. 
ción, por iniciativa del Dr. Ennio Vivaldi, en Chile la ergonomía es una disciplina que está en un estado bastante inicial de desarrollo y, lamentablemente, en forma habitual se la circunscribe a aspectos parciales, principalmente relacionados con posturas inadecuadas o trabajo repetitivo que, siendo muy importantes, no se pueden tratar como temas aislados del sistema global de trabajo. Por eso es importante analizar qué es y qué aporta la ergonomía para que los trabajadores, realizando actividades eficientes, tengan una buena calidad de vida laboral.

El término ergonomía deriva de dos palabras griegas: ergo (trabajo) y nomos (leyes, reglas). Por lo tanto, en el estricto sentido de la palabra, significa leyes o reglas del trabajo. Fue introducida en 1949 por el psicólogo británico K.F.H. Murrell, cuando un grupo de científicos se reunió en Inglaterra para formar la Sociedad de Investigaciones Ergonómicas. La idea fue cobijar bajo el mismo alero a ingenieros, fisiólogos, anatomistas, psicólogos, higienistas industriales, arquitectos, profesionales del área de la salud y en general personas interesadas en el comportamiento humano en el trabajo. Murrell (1969), en su clásico libro denominado Ergonomics, señala la razón que lo llevó a proponer la palabra ergonomía. Él plantea que es simple, que se puede traducir a cualquier idioma y, lo más importante, que no otorga preponderancia a ninguna especialidad en particular, lo que resalta su carácter multidisciplinario.

La Sociedad de Ergonomía define esta disciplina como el estudio científico del hombre en su trabajo; en particular la aplicación de conceptos de anatomía, fisiología y psicología humanas en el diseño del trabajo. Con el correr del tiempo, algunos especialistas en el tema han considerado que esta definición es restrictiva y han propuesto otras más de acuerdo con su carácter interdisciplinario. Entre ellos, Zander (1986) señala que "la ergonomía es el estudio del hombre en el trabajo, con el propósito de lograr un óptimo sistema hombre-tarea, en el cual pueda mantenerse un adecuado balance entre el trabajador y las condiciones laborales". En otras palabras, la ergonomía es una disciplina que actúa como un puente entre la biología humana y la ingeniería, poniendo a disposición de ésta última conocimientos de las capacidades y limitaciones humanas que deben ser utilizados para un buen diseño del trabajo.

\section{IMPORTANCIA DE LA VISIÓN ERGONÓMICA DEL TRABAJO}

Los objetivos de la ergonomía son promover la salud y el bienestar, reducir los accidentes y mejorar la productividad de las empresas. Al observar los objetivos enumerados, cabe la pregunta: ¿aporta algo nuevo la ergonomía? No hay duda que la seguridad industrial al igual que la medicina del trabajo se han preocupado de la salud y la prevención de accidentes, alcanzando un alto grado de desarrollo en este siglo. Lo mismo puede afirmarse con relación a la psicología del trabajo, cuyos métodos están orientados a fomentar el bienestar de los trabajadores. Por otra parte, con relación a la eficiencia, desde la época de la revolución industrial hasta el actual desarrollo de la ingeniería industrial, se ha avanzado notablemente en la búsqueda de métodos que permitan aumentar la cantidad y calidad de los productos. Por ello que la pregunta es válida. No obstante, la ergonomía tiene un carácter integrativo y anticipativo, ya que tiende a crear herramientas, máquinas, puestos de trabajo y métodos que se adapten a las capacidades y limitaciones humanas. En otras palabras, cuando se diseña cualquier actividad en que tend rá participación el hombre, es cuando debe evaluarse los efectos que dichos elementos podrían tener sobre quien interactúa con ellos. No hay que olvidar que desde una simple herramienta manual hasta los más complejos sistemas industriales, son creados por seres humanos para ayudarse en el cumplimiento de sus tareas. Si, por ejemplo, pudiéramos volar a la velocidad de un avión, sin sufrir efectos adversos de la altura, clima, etc., no necesitaríamos aviones para desplazarnos grandes distancias. Por ello, resulta una paradoja que 
no se preste atención a los elementos de uso humano hasta que estos empiezan a crear daños en la población o a demostrar ineficiencia.

En Chile, al igual que en muchos otros países, hay dos grandes aspectos del trabajo en que la ergonomía puede hacer importantes contribuciones. Uno de ellos se refiere a los problemas de adaptación a trabajos manuales pesados. En este tipo de tareas, el hombre, utilizando simples herramientas, aporta la parte más importante de la energía requerida para el cumplimiento de una determinada función. Esto puede ser bastante crítico, particularmente cuando hay factores agregados como, por ejemplo, el calor.

El segundo aspecto, igualmente comple- jo, es el trabajo mecanizado. En la últimas décadas se observan grandes cambios en las formas tradicionales de producción. La energía humana se reemplaza por maquinarias, que son capaces de hacer cantidades mucho mayores de trabajo que cualquier ser humano. Esto hace que los trabajadores sean progresivamente más sedentarios, limitando sus acciones a percibir información, interpretarla y a ejecutar sus decisiones para mantener o cambiar el curso de algún proceso, con acciones musculares livianas, pero con una alta participación de los procesos mentales. En ambos tipos de tareas, la ergonomía tiene procedimientos de estudio diferentes, pero basados en una raíz común, que se ilustra en la Figura 1.

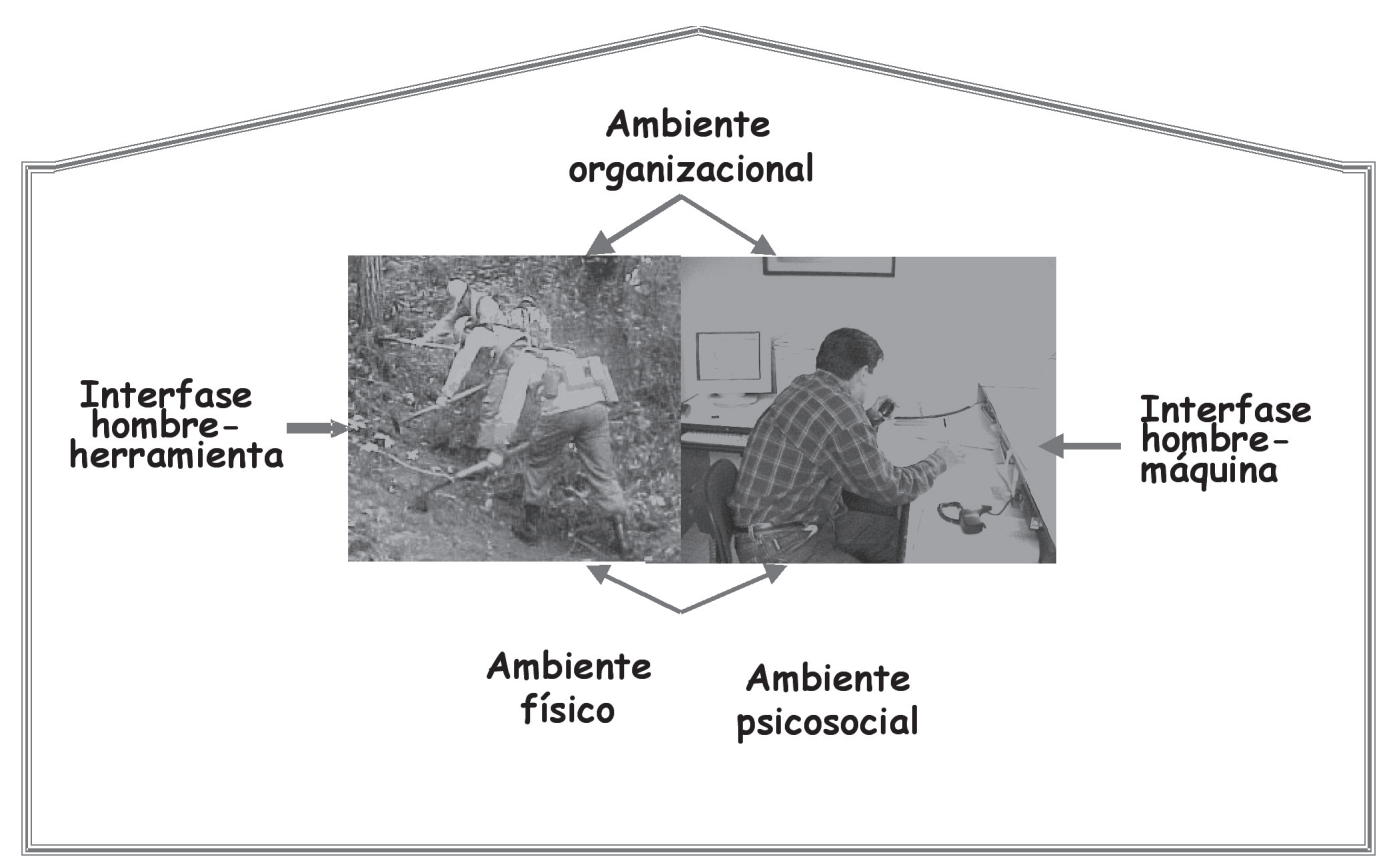

Figura 1. Visión ergonómica del trabajo.

En la figura se puede ver, para dos actividades tan disímiles como construir cortafuegos en incendios forestales con herramientas manuales y trabajar en una sala de control computarizada, que el hombre percibe información de su entorno laboral, toma decisiones y las ejecuta mediante acciones me- cánicas. Si bien el esquema básico es el mismo, las exigencias del trabajo son completamente diferentes; mientras las decisiones del trabajador que emplea herramientas manuales son simples, su respuesta mecánica puede ser causal de trabajo físico pesado. En cambio, en el caso de la sala de control, el trabajo 
físico es mínimo, pero la complejidad en la toma de decisiones es significativamente mayor que en el caso anterior. En todo caso, independiente del tipo de tarea, el hombre está en un sistema de retroalimentación constante con su trabajo, en el cual percibe información, la interpreta, toma decisiones y efectúa alguna acción para continuar con el curso del proceso. El diseño ergonómico debe permitirle cumplir cada etapa en forma segura y eficiente, sea su actividad manual o mecanizada.

Lo anterior corresponde a la relación directa entre el hombre y su tarea. Sin embargo, los trabajos se realizan en lugares físicos donde el ser humano puede sufrir los efectos del calor, frío, ruido, vibraciones mecánicas, etc. Estos agentes, cuando exceden límites recomendables alteran el bienestar, pudiendo producir también problemas de salud física y mental, que influyen en el accionar de un trabajador. Muchos de ellos provienen de las propias máquinas, como el ruido y las vibraciones, pero también pueden tener su origen en la naturaleza, por ejemplo, el frío y el calor. En algunos casos, aunque estos agentes se mantengan bajo niveles que puedan provocar enfermedad, pueden ser causales de estrés ocupacional y llegar a producir problemas psicológicos o sobrecarga fisiológica y fatiga en los trabajadores.

Lo destacado en los párrafos precedentes engloba los problemas inherentes a las estaciones de trabajo y el ambiente físico, pero hay muchos otros factores que no dependen del lugar de trabajo en sí, sino que de la organización del sistema o proceso en que la actividad está inserta. De manera que el concepto moderno de ergonomía considera el análisis de las actividades de un trabajador como parte de un sistema que en su conjunto debe ser eficientemente diseñado. Los procesos industriales tienen una entrada y una salida, pasando por varias estaciones intermedias. En las distintas etapas de un proceso, hay seres humanos que cumplen tareas específicas, y sólo la adaptación de cada persona y de todas ellas como conjunto permitirá el mejor aprovechamiento de los recursos, tanto humanos como materiales, sin poner en riesgo a las personas. Esta forma de aproximación puede ser altamente eficiente, en la medida que los problemas de trabajo humano se aborden en forma multidisciplinaria, con un sentido de equipo en el cual los profesionales de la salud tienen mucho que aportar, ya que son precisamente los que pueden ver frustrados sus esfuerzos curativos si los agentes causales de los síntomas persisten en el medio laboral.

\section{PROBLEMAS ERGONÓMICOS ACTUALES Y FUTUROS}

En Chile, al igual que en el resto de América Latina, una parte importante de la población subsiste en base a su propio esfuerzo muscular accionando herramientas de bajo costo, de corta vida útil y que, por ser cambiadas frecuentemente, pueden mejorarse y reemplazarse sin que las empresas deban incurrir en grandes inversiones. Este es un tema no superado y en el que la ergonomía tiene aún mucho que aportar, ya que se puede reducir riesgos de problemas músculoesqueléticos, fatiga y accidentes, si se mejora la organización del trabajo y se adaptan herramientas y accesorios a sus características. Sin embargo, no basta sólo con esto, sino que también se requiere considerar otros aspectos tales como alimentación, provisión de elementos de seguridad adecuados, capacitación y exigencias de rendimiento que no sobrepasen límites recomendables de esfuerzo físico. La adaptación ergonómica de los trabajos manuales no es fácil, pero con estudios sistemáticos se puede ir motivando a los empresarios para introducir cambios simples que incrementan el bienestar de sus trabajadores.

El ejemplo más claro es el desarrollo alcanzado en el sector forestal chileno, que ha sido ampliamente analizado en dos libros publicados por la Organización Internacional del Trabajo, OIT, (Apud y cols. 1989, 1995). Los primeros estudios en el campo forestal se orientaron a mejorar las condicio- 
nes de vida en los campamentos, que son los hogares temporales para estos trabajadores. Los dormitorios, áreas de recreación y comedores que habitualmente usaban estaban lejos de las condiciones mínimas de higiene y comodidad. Lo mismo ocurría con la alimentación, que en trabajos manuales de alta intensidad debe ser aportada en cantidad suficiente para equilibrar los requerimientos calóricos y de nutrientes que demandan estas tareas.

Cumplidos los requisitos básicos de vivienda, alimentación y recreación, los trabajadores requieren ser capacitados, siendo ésta la instancia precisa para reforzar técnicas seguras de trabajo. Las etapas descritas son una condición previa a la adaptación ergonómica de herramientas, técnicas y métodos de trabajo. Los resultados hasta ahora obtenidos han sido francamente auspiciosos. Los criterios aplicados en el estudio de trabajos físicos pesados han permitido superar lo meramente descriptivo. En otras palabras, la orientación no ha estado sólo en seguir cuantificando cuántos accidentes se producen, cuáles son las deficiencias de alimentación o de vivienda en los campamentos, etc., sino que, sobre la base de los conocimientos generados con criterios multidisciplinarios, se han buscado soluciones para superarlos con la participación de empresarios y trabajadores.

Lo que se ha señalado para trabajos manuales tradicionales, es también válido para la innovación tecnológica del futuro. A menudo, cuando se planifica la mecanización de un sistema de trabajo existe bastante claridad respecto a costos y niveles de producción esperables, pero son escasas, por no decir nulas, las ocasiones en que los planificadores pueden responder sobre los efectos que dichos sistemas tendrán sobre la seguridad y salud de los trabajadores. Como el costo de la mecanización es alto, cuando las maquinarias se adquieren sin pensar en los operadores, si su diseño es inadecuado, los trabajadores deben continuar expuestos a los riesgos hasta que la máquina termine por destruirse. Modificar estos elementos para reducir problemas tangibles derivados, por ejemplo, de la postura de trabajo, la repetitividad de las acciones, la visibilidad de los equipos, el diseño de controles e indicadores, el ruido, las vibraciones, etc. rara vez se realizan, con consecuencias directas para la población laboral, que se manifiestan en niveles críticos de accidentabilidad, deterioro de la salud y subutilización de las maquinarias por la inadecuada adaptación de ellas a los usuarios. Mientras esto siga ocurriendo parece improcedente hablar de desarrollo tecnológico y más bien debería mantenerse el término mecanización. Desde un punto de vista ergonómico, el desarrollo tecnológico sólo se consigue en la medida que la mecanización, además de producir aumentos en la producción, no presente riesgos para los trabajadores. Cuando esto no se logra, el recurso humano aparece como un término abstracto desconectado de los elementos que lo integran que son personas. Esta es una barrera que es necesario superar y, a nuestro juicio, la ergonomía puede contribuir porque, por definición, propende a un trabajo multidisciplinario donde cada integrante del equipo tiene algo que aportar en materias específicas, que ayuden al logro del objetivo central que es la protección del hombre. Por esta razón, la ergonomía del futuro requerirá cada vez más que los profesionales de la salud incorporen en sus mallas curriculares conceptos sobre problemas de adaptación del trabajo al hombre.

Esta es una breve síntesis de los propósitos de la ergonomía. En el fondo se puede sostener que esta disciplina aporta criterios integrativos para el análisis y solución de los problemas que afectan al hombre en su trabajo. La ergonomía tiene un carácter "constructivo" que es fundamental para la incorporación de tecnologías "sanas". Las labores correctivas, por lo general, son de alto costo y nunca permiten soluciones completamente satisfactorias. También es importante la disposición de las empresas para realizar intervenciones ergonómicas. Un mejor ambiente laboral, que ayude a mantener el estado de alerta y la motivación, como por ejemplo, 
creando mayor variación en las tareas, haciendo el ambiente más estimulante e incorporando a los trabajadores en la toma de decisiones respecto a la mejor forma de realizar sus actividades, son elementos fundamentales en la búsqueda de sistemas de trabajo en que se equilibre bienestar humano y productividad.

Estos principios tan lógicos son difíciles de llevar a la práctica por diversas razones. Una de las más importantes es que no existe un especialista único capaz de entender y dar solución a los múltiples y diferentes problemas laborales. En este contexto, es necesario hacer una diferenciación entre lo que podríamos denominar estudios básicos, estudios ergonómicos aplicados y aplicaciones propiamente tales. Si se considera que el diseño ergonómico del trabajo apunta al buen uso de las capacidades del hombre con respeto por sus limitaciones, es necesario investigar para conocer con la mayor certeza los límites humanos y así realizar acciones para evitar que el trabajo sobrepase las capacidades física y mental de los trabajadores. Por otra parte, el puente entre el conocimiento básico y las aplicaciones lo constituyen las investi- gaciones ejecutadas con fines correctivos en actividades ya existentes y los estudios anticipativos realizados en el estado de planificación de un trabajo. Es en estas etapas donde los profesionales de la salud pueden contribuir con conocimientos específicos que ayudan a una mejor comprensión de los problemas y a la búsqueda de soluciones prácticas. Por último, la acción final que implica implementar sistemas de trabajo basados en resultados de los estudios multidisciplinarios previos, es una decisión que toman los empresarios y, principalmente, los ingenieros involucrados en los procesos productivos.

\section{BIBLIOGRAFÍA}

APUD, E., BOSTRAND, L., MOBBS, Y. y B. STREHLKE (1989). Guidelines on ergonomic study in forestry. Ed.: Organización Internacional del Trabajo (OIT), Ginebra.

APUD, E. y VALDÉS, S. (1995). Ergonomics in Forestry: The Chilean Case. Ed.: Organización Internacional del Trabajo (OIT), Ginebra.

MURREL, K.F.H. (1969). Ergonomics. Ed.: Chapman and Hall, Inglaterra.

ZANDER, J. (1986). Introduction to Ergonomics. Documentos del Curso Internacional de Ergonomía, Wageningen. 